\title{
Special issue on multigrid methods
}

\author{
Artem Napov $^{1} \cdot$ Yvan Notay $^{1} \cdot$ Stefan Vandewalle $^{2}$
}

Accepted: 18 December 2015 / Published online: 6 January 2016

(C) Springer-Verlag Berlin Heidelberg 2016

This special issue of Computing and Visualization in Science contains selected papers from the 2014 European Multigrid conference (EMG 2014), which took place in Leuven, Belgium, from 9 to 12 September, 2014.

European Multigrid (EMG) is a series of conferences on the theme of multigrid methods and related fields. EMG is one of the most important conference series on this topic worldwide. Previous EMG meetings have been held in Cologne (1981 and 1985), Bonn (1990), Amsterdam (1993), Stuttgart (1996), Gent (1999), Hohenwart (2002), Scheveningen (2005), Bad Herrenhalb (2008), Ischia (2010) and Schwetzingen (2012).

The EMG 2014 conference took place at the Irish College, in Leuven, an old university college founded in 1607. It attracted more than 80 participants of 15 countries, of which 20 were PhD-students; 12 participants attended the conference from outside Europe. Further details are available on the conference homepage

\section{http://metronu.ulb.ac.be/EMG2014/}

The contributions presented at EMG 2014 provided a snapshot of the research activities in multigrid methods and related fields. They demonstrated that some of the wellestablished topics, such as algebraic multigrid, non-linear

Artem Napov

anapov@ulb.ac.be

Yvan Notay

ynotay@ulb.ac.be

Stefan Vandewalle

Stefan.Vandewalle@cs.kuleuven.be

1 Service de Métrologie Nucléaire, Université Libre de Bruxelles (C.P. 165/84), Brussels, Belgium

2 Department of Computer Science, KU Leuven - University of Leuven, Leuven, Belgium multigrid, parallel multigrid, Fourier analysis, multi-level optimization, and multigrid for CFD applications, still represent an active area of research. The talks also addressed more recent topics, such as parallel-in-time multigrid, multigrid for the Helmholtz equation, structured solvers and preconditioners (including $\mathscr{H}$ - and $\mathscr{H}^{2}$-matrices), as well as some emerging trends, including application to biology. This diversity of topics is further reflected in the papers selected for the present special issue.

This special issue will appear in two volumes. The first volume contain three papers [1-3]. Hackbusch [1] gives an overview of various tensor-based methods for the solution of linear systems. Kriemann and Le Borne [2] study some factored approximate inverses based on $\mathscr{H}$-matrix arithmetics. Speleers and coauthors [3] prove $h$-optimality of two-grid methods for B-spline isogeometric analysis discretizations.

Acknowledgments We would like to acknowledge the financial support of FWO (Fonds Wetenschappelijk Onderzoek-Vlaanderen), FNRS (Fonds de la Recherche Scientifique-FNRS) and CWI (Centrum Wiskunde \& Informatica). We also would like to thank Irish Collegethe conference venue-for their efforts and hospitality. Last but not least, we are grateful to Dirk Abbeloos and Stephanie Friedhoff for their valuable assistance during the conference.

\section{References}

1. Hackbusch, W.: Solution of linear systems in high spatial dimensions. Comput. Vis. Sci. 17(3) (2016). doi:10.1007/ s00791-015-0252-0

2. Kriemann, R., Le Borne, S.: H-FAINV: Hierarchically factored approximate inverse preconditioners. Comput. Vis. Sci. 17(3) (2016). doi:10.1007/s00791-015-0254-y

3. Donatelli, M., Garoni, C., Manni, C., Serra-Capizzano, S., Speleers, H.: Two-grid optimality for Galerkin linear systems based on B-splines. Comput. Vis. Sci. 17(3) (2016). doi:10.1007/ s00791-015-0253-z 